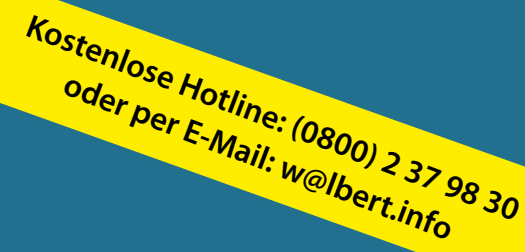

Leser der MMW können sich mit allen Fragen zur Abrechnung und Praxisführung an Helmut Walbert, Facharzt für Allgemeinmedizin, Würzburg, wenden. Sie erreichen inn jeden Donnerstag von 13 bis 15 Uhr unter der kostenlosen Rufnummer (0800) 2379830 oder per E-Mail: w@lbert.info.

Helmut Walbert

Allgemeinarzt, Medizinjournalist und Betriebswirt Medizin

\title{
Wie rechne ich ab, wenn ich dort am selben Tag mehrere Patienten betreue?
}

Dr. med. S. I., hausärztlicher Internist, Kiel: Wie rechne ich Heimbesuche ab, wenn ein Pflegheim für mehrere Patienten schriftlich Hausbesuche noch am gleichen Tag anfordert?

Antwort: Der erste Besuch wird auf jeden Fall nach GOP 01 415, „Dringender Besuch eines Patienten in beschützenden Wohnheimen bzw. Einrichtungen bzw. Pflege- oder Altenheimen mit Pflegepersonal wegen der Erkrankung, noch am Tag der Bestellung ausgeführt; 54,15 €" abgerechnet.

Die GOP 01415 ist im Rahmen des organisierten Not(-fall)dienstes nicht berechnungsfähig und nicht neben den GOP 01100 bis 01 102, sowie anderen Besuchen. Weitere einschränkende Bestimmungen

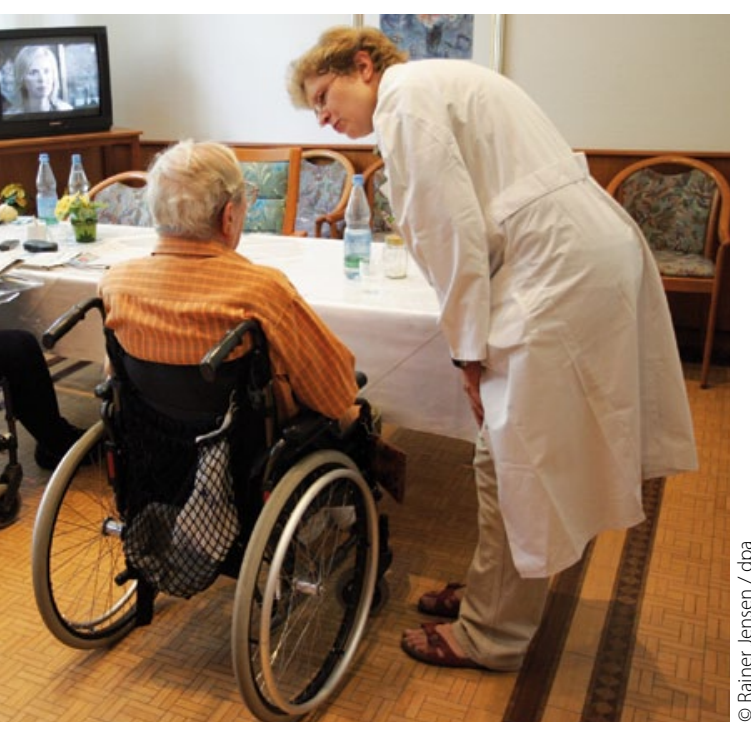

Die „soziale Gemeinschaft" spielt bei der Abrechnung eine entscheidende Rolle. finden sich im Text der Gebührenordnung nicht. Somit wären ein weiterer dringender Besuch „Herr Doktor, Sie sollen bitte auch noch in der Gruppe XY einen dringenden Besuch machen" ebenfalls nach GOP 01415 abrechenbar. Alle weiteren Besuche innerhalb einer Wohngruppe sind nach GOP 01413 abzurechnen. Am Samstag zwischen 7 und 14 Uhr kann zusätzlich abgerechnet werden die GOP 01 102, ,Inanspruchnahme des Vertragsarztes an Samstagen zwischen 7.00 und 14.00 Uhr, 9,99 €".

Der angesehene Kommentar Wezel/Liebold, der von einigen KVen zur Grundlage der Abrechnungsbestimmungen genommen wird, führt allerdings aus: „Die GOP 01415 kann im Rahmen einer Besuchstätigkeit in einer der genannten Einrichtungen grundsätzlich nur einmal abgerechnet werden. Die Besuche bei weiteren Kranken sind nach der Gebührenordnungsposition 01413 abzurechnen, und zwar auch dann, wenn es sich hierbei ebenfalls um dringende Besuche handelt."

Hier wird meines Erachtens zu restriktiv und nicht mehr zeitgemäß interpretiert. Insbesondere die Interpretation „derselben sozialen Gemeinschaft" kann so nicht mehr akzeptiert werden. Der Kommentar:„Der Begriff,soziale Gemeinschaft' ist weiträumig. Er erfasst nicht nur die zusammen wohnenden Angehörigen einer Familie, sondern auch sämtliche Bewohner eines Altenheimes sowie anderer Heime (z.B. Kinderheime, Kur- und Ferienheime, Kasernen, Lager, Pflegeheime), sofern ein Gemeinschaftsleben z. B. regelmäßige gemeinsame Einnahme aller oder fast aller Mahlzeiten oder Versorgung mit Essen in den Zimmern - gepflegt wird."

Diese Interpretation geht an der heutigen Organisationstruktur modern geführter Altenheime mit kleinen voneinander unabhängig geführten "sozialen Gemeinschaften" vorbei. Hier trifft der Arzt von Wohngemeinschaft zu Wohngemeinschaft auf unabhängig voneinander arbeitende Teams und von anderen Wohngemeinschaften unabhängig lebende „Mitbewohner" der gleichen Einrichtung. Der Aufwand ist gleich einem Erstbesuch. Deshalb liegen hier meines Erachtens die Kriterien eigenständiger sozialer Gemeinschaften vor und somit die Berechtigung zum Abrechnen eines weiteren Besuches.

\section{So sind Sie auf der sicheren Seite: \\ Besuch sauber dokumentieren}

Meine Empfehlung lautet, die Hausbesuche je nach Struktur des Altenheims abzurechnen. Es sollte insbesondere bei zusätzlichen dringenden Besuchen nach GOP 01415 sauber dokumentiert werden nach folgenden Punkten: Zeitpunkt der Bestellung des Besuchs / die Kriterien, aus denen das Personal die Dringlichkeit abgeleitet hat / die beim Patienten erhobenen Befunde, sowie die therapeutischen Maßnahmen. Entscheidend für die Dringlichkeit ist die subjektive Einschätzung durch das Personal, nicht das Ergebnis der Untersuchung. Wichtig: Wegegebühren sind allerdings nur einmal pro Anfahrt abrechenbar. 